\title{
THE ASYMPTOTIC BEHAVIOR OF GAS IN AN $n$-DIMENSIONAL POROUS MEDIUM ${ }^{1}$
}

BY

\author{
AVNER FRIEDMAN AND SHOSHANA KAMIN
}

\begin{abstract}
Consider the flow of gas in an $n$-dimensional porous medium with initial density $u_{0}(x) \geqslant 0$. The density $u(x, t)$ then satisfies the nonlinear degenerate parabolic equation $u_{t}=\Delta u^{m}$ where $m>1$ is a physical constant. Assuming that $I \equiv \int u_{0}(x) d x<\infty$ it is proved that $u(x, t)$ behaves asymptotically, as $t \rightarrow \infty$, like the special (explicitly given) solution $V(|x|, t)$ which is invariant by similarity transformations and which takes the initial values $\delta(x) I(\delta(x)=$ the Dirac measure) in the distribution sense.
\end{abstract}

1. Statement of the main result. Consider the Cauchy problem for $u(x, t)$ :

$$
\begin{aligned}
u_{t} & =\Delta u^{m} \quad\left(x \in R^{n}, t>0\right), \\
u(x, 0) & =u_{0}(x) \quad\left(x \in R^{n}\right) .
\end{aligned}
$$

The function $u$ represents the density of a gas in a porous medium and $m$ is a physical constant, $m>1$. We assume that

$$
\begin{aligned}
& u_{0}(x) \text { is continuous, } u_{0}(x) \geqslant 0, \quad u_{0}(x) \neq 0, \quad u_{0}(x) \leqslant M, \\
& u_{0} \in L^{1}\left(R^{n}\right) \cap L^{2}\left(R^{n}\right) \quad(M \text { constant }),
\end{aligned}
$$

and set

$$
I=\int_{R^{n}} u_{0}(x) d x
$$

By a weak solution of (1.1), (1.2) we mean a function $u$ satisfying, for any $T>0$,

$$
\int_{0}^{T} \int_{R^{n}}\left[(u(x, t))^{2}+\left|\nabla_{x} u^{m}(x, t)\right|^{2}\right] d x d t<\infty
$$

and

$$
\int_{0}^{T} \int_{R^{n}}\left(u \frac{\partial f}{\partial t}-\nabla_{x} u^{m} \cdot \nabla_{x} f\right) d x d t+\int_{R^{n}} u_{0}(x) f(x) d x=0
$$

for any continuously differentiable function $f$ with compact support in $R^{n} \times[0, T)$.

It is well known [14], [11] that given $u_{0}$ satisfying (1.3), (1.4) there exists a unique generalized solution $u$ of (1.1), (1.2) and

$$
\int_{R^{n}} u(x, t) d x=I \text { for all } t>0
$$

Received by the editors August 27, 1979.

AMS (MOS) subject classifications (1970). Primary 35K55, 76S05; Secondary 35K 15.

${ }^{1}$ This work is partially supported by National Science Foundation Grant MCS-7817204 and AFOSR Grant 78-3602. 
a very general uniqueness theorem was recently proved by Brezis and Crandall [4]. By a recent result of Caffarelli and Friedman [5], [6] $u(x, t)$ is Hölder continuous in $(x, t)$ uniformly in any strip $t \geqslant \delta(\delta>0)$.

In this paper we are interested in the asymptotic behavior of $u(x, t)$ as $t \rightarrow \infty$. To state the main result we first introduce the similarity solution $V_{L}$ of Barenblatt and Prattle [13]. Let

$$
G(s)=\left[\left(\beta^{2}-c^{2} s^{2}\right)^{+}\right]^{1 /(m-1)}
$$

where

$$
c^{2}=\frac{l(m-1)}{2 m n}, \quad l=\frac{1}{m-1+2 / n}
$$

and $\beta$ is a positive constant such that $\int_{R^{n}} G(|x|) d x=1$. Then, for any $L>0$,

$$
V_{L}(r, t)=L^{1 /(m-1)} \frac{1}{L^{l} t^{l}} G\left(\frac{r}{L^{l / n} t^{l / n}}\right), \quad r=|x|,
$$

is a solution of

$$
\frac{\partial}{\partial t} V_{L}=\Delta\left(V_{L}\right)^{m}
$$

satisfying $V_{L}(r, 0)=\delta(x) L^{1 /(m-1)}(\delta(x)=$ the Dirac measure) in the sense of distributions. As easily verified,

$$
\int_{R^{n}} V_{L}(r, t) d x=L^{1 /(m-1)}
$$

We can now state the main result of the paper.

THEOREM 1.1. As $t \rightarrow \infty$,

$$
t^{\prime}\left|u(x, t)-V_{L_{0}}(r, t)\right| \rightarrow 0
$$

uniformly with respect to $x$ in any set $|x| \leqslant C t^{l / n}(C>0)$, where

$$
L_{0}=I^{m-1} \text {. }
$$

For $n=1$ this theorem was proved by Kamin (Kamenomostkaya) [9], [10]. The proof for $n \geqslant 1$ given in this paper employs a different approach than in [9], [10] and exploits the continuity of the solution $u$; both methods use similarity transformations.

For $n=1$ Peletier [12] and Van Duyan and Peletier [7] studied the asymptotic behavior in a half-plane $x>0$ and in the whole space when $u_{0}(x) \sim A$ as $x \rightarrow \infty$, $u_{0}(x) \sim B$ as $x \rightarrow-\infty ; A>0, B>0$. For a bounded domain $\Omega$ in $\mathbf{R}^{n}$, Aronson and Peletier [1] have recently obtained the asymptotic behavior of the solution in $\{(x, t) \in \Omega \times(0, \infty)\}$; they assume that $u(x, t)=0$ if $x \in \partial \Omega$. Both the result and method are different from those obtained in the present work.

To facilitate the reading of the proof, which takes the rest of the paper, we have broken it into several parts. In $\$ 2$ we derive some properties of the functions $V_{L}$. In $\S 3$ we study the function $L(t)$, where $L(t)$ is defined as the largest value of $L$ such that, for some $\tau \geqslant 0$,

$$
V_{L}(r, t+\tau) \leqslant u(x, t) \quad\left(\text { for all } x \in R^{n}\right)
$$


In $\$ 4$ we prove Theorem 1.1 in case $u_{0}(x)$ has compact support and, finally, in $\$ 5$ we give the proof in the general case.

2. Auxiliary lemmas on $V_{L}$. Notice that

$$
\text { support of the function } r \rightarrow V_{L}(r, t) \text { is given by } r \leqslant \frac{L^{l / n} t^{l / n}}{c / \beta} \text {. }
$$

LEMMA 2.1. For fixed $L>0, t>0$, if $\varepsilon>0$ is sufficiently small then

$$
\begin{aligned}
& V_{L}(r, t-\varepsilon)>V_{L}(r, t) \text { for } 0<r<\theta \frac{L^{l / n} t^{l / n}}{c / \beta}, \\
& V_{L}(r, t-\varepsilon)<V_{L}(r, t) \text { for } \theta \frac{L^{l / n} t^{l / n}}{c / \beta}<r<\frac{L^{l / n} t^{l / n}}{c / \beta}
\end{aligned}
$$

where

$$
\theta=\theta_{\varepsilon} \rightarrow \theta_{0} \quad \text { as } \varepsilon \rightarrow 0 ; \quad \theta_{0}=\sqrt{(m-1) l}
$$

Observe that

$$
0<\theta_{0}<1 \text {. }
$$

Proof. We solve, for small $\varepsilon$, the equation in $r$ :

$$
V_{L}(r, t-\varepsilon)=V_{L}(r, t) \text {. }
$$

Thus

$$
\left(\frac{t-\varepsilon}{t}\right)^{l(m-1)} G^{m-1}\left(\frac{r}{L^{l / n} t^{l / n}}\right)=G^{m-1}\left(\frac{r}{L^{l / n}(t-\varepsilon)^{l / n}}\right) .
$$

By the mean value theorem the right-hand side is equal to

$$
G^{m-1}\left(\frac{r}{L^{l / n} t^{l / n}}\right)+\frac{r}{L^{l / n}}\left(\frac{1}{(t-\varepsilon)^{l / n}}-\frac{1}{t^{l / n}}\right)\left(G^{m-1}\right)^{\prime}\left(\frac{r}{L^{l / n} \tilde{t}^{l / n}}\right)
$$

where $t-\varepsilon<\tilde{t}<t$; also

$$
\begin{aligned}
\left(G^{m-1}\right)^{\prime}(s) & =-2 c^{2} s \\
\left(\frac{t-\varepsilon}{t}\right)^{l(m-1)} & \sim 1-\frac{l(m-1)}{t} \varepsilon \\
\frac{1}{(t-\varepsilon)^{l / n}}-\frac{1}{t^{l / n}} & =-\frac{1}{t^{l / n}}\left(1-\left(\frac{t}{t-\varepsilon}\right)^{l / n}\right) \sim \frac{1}{t^{l / n}} \frac{l / n}{t} \varepsilon .
\end{aligned}
$$

Using these facts in (2.5), we get

$$
\frac{l(m-1)}{t} c \varepsilon G^{m-1}\left(\frac{r}{L^{l / n} t^{l / n}}\right) \sim 2 \frac{c^{2} r^{2}}{L^{2 l / n} t^{2 l / n}} \frac{l / n}{t} \varepsilon,
$$

so that

$$
n(m-1)\left(\beta^{2}-\frac{c^{2} r^{2}}{L^{2 l / n} t^{2 l / n}}\right) \sim \frac{2 c^{2} r^{2}}{L^{2 l / n} t^{2 l / n}}
$$


It follows that the solution $r$ of (2.4) is given by

$$
\theta \frac{\beta}{c} L^{l / n} t^{l / n}
$$

where

$$
\theta=\theta_{\varepsilon} \rightarrow\left(\frac{n(m-1)}{n(m-1)+2}\right)^{1 / 2}=\theta_{0} \quad \text { as } \varepsilon \rightarrow 0,
$$

and the proof of the lemma follows.

Lemma 2.2. For any $x^{0} \in R^{n}$ and $\tau$ real,

$$
t^{\prime}\left|V_{L}\left(\left|x-x^{0}\right|, t+\tau\right)-V_{L}(|x|, t)\right| \rightarrow 0 \text { as } t \rightarrow \infty,
$$

uniformly with respect to $x \in R^{n}$.

The proof is immediate from (1.6).

Choose a point $y^{0} \in R^{n}$ such that $u_{0}\left(y^{0}\right)>0$; then, for some $\delta_{0}>0, \eta_{0}>0$,

$$
u_{0}(x) \geqslant \delta_{0} \quad \text { if }\left|x-y^{0}\right|<\eta_{0} \text {. }
$$

But then we can find $L_{1}>0, \tau_{1}>0$ such that

$$
u_{0}(x) \geqslant V_{L_{1}}\left(\left|x-y^{0}\right|, \tau_{1}\right) \text { for all } x \in R^{n} \text {. }
$$

Indeed, we simply have to choose

$$
L_{1} \tau_{1}=c_{1}, \quad c_{1} \text { sufficiently small, }
$$

and then choose $L_{1}$ sufficiently small (depending on $c_{1}$ ).

If we prove that

$$
t^{\prime}\left|u(x, t)-V_{L_{0}}\left(\left|x-y^{0}\right|, t\right)\right| \rightarrow 0 \text { as } t \rightarrow \infty,
$$

uniformly in $x \in R^{n}$ in any set $|x| \leqslant C t^{l / n}$, then, in view of Lemma 2.2 , the assertion of Theorem 1.1 would follow.

For simplicity of notation we fix the origin at the point $y^{0}$. Thus (2.7) becomes

$$
u_{0}(x) \geqslant V_{L_{1}}\left(r, \tau_{1}\right), \quad r=|x|,
$$

and the assertion (2.8) reduces to the assertion (1.8). From (2.9) it follows, by comparison, that

$$
u(x, t) \geqslant V_{L_{1}}\left(r, t+\tau_{1}\right) \quad\left(x \in R^{n}, t>0\right) .
$$

From now on, until the end of $\S 4$, we impose the restriction:

$$
u_{0} \text { has compact support. }
$$

We can then find $L_{2}>0, \tau_{2}>0$ such that

$$
u_{0}(x) \leqslant V_{L_{2}}\left(r, \tau_{2}\right) \text { for all } x \in R^{n} .
$$

Indeed, we simply take first $L_{2} \tau_{2}=c_{2}$ where $c_{2}$ is sufficiently large and then choose $L_{2}$ to be sufficiently large, depending on $c_{2}$.

From (2.12), we deduce that

$$
u(x, t) \leqslant V_{L_{2}}\left(r, t+\tau_{2}\right) \quad\left(x \in R^{n}, t>0\right) .
$$


Suppose for some $t_{0}>0, \tau$ real, $t_{0}+\tau>0$,

$$
u\left(x, t_{0}\right) \equiv V_{L}\left(r, t_{0}+\tau\right) .
$$

Then, by uniqueness,

$$
u(x, t)=V_{L}(r, t+\tau) \text { if } t>t_{0} .
$$

Recalling Lemma 2.2, the assertion of Theorem 1.1 then follows. Thus, in order to prove Theorem 1.1 (under the condition (2.11)), we may assume, without loss of generality, that

$$
\begin{aligned}
& \text { for any } L>0, \quad \tau \text { real, } \quad t>0, \quad t+\tau>0, \\
& u(x, t) \not V_{L}(r, t+\tau) \quad\left(x \in R^{n}\right) .
\end{aligned}
$$

For any fixed $t>0$, denote by $\Sigma_{t}$ the set of all points $(L, \tau)$ such that $L>0$, $\tau \geqslant 0$, and

$$
u(x, t) \geqslant V_{L}(r, t+\tau)
$$

and set

$$
L(t)=\sup _{(L, \tau) \in \Sigma_{t}} L
$$

\section{Properties of $L(t)$.}

LEMMA 3.1. There exists a point $\left(L^{*}, \tau^{*}\right) \in \Sigma_{t}$ such that $L(t)=L^{*}$ and

$$
\tau^{*} \leqslant C(t+1)
$$

$C$ is a constant independent of $t$.

Proof. The inequality (2.16) implies

$$
\int_{R^{n}} V_{L}(r, t+\tau) d x \leqslant \int_{R^{n}} u(x, t) d x=I
$$

where (1.5) was used. Recalling (1.7) we conclude that $L \leqslant I^{m-1}$. From (2.10) we also deduce that $L \geqslant L_{1}$. Thus, in seeking to find $\sup L$ in (2.17) we may restrict the $L$ to lie in the interval

$$
L_{1} \leqslant L \leqslant I^{m-1}
$$

In view of (2.16) and (3.2),

$$
\text { support of } x \rightarrow u(x, t) \text { contains the set } r \leqslant C_{1}(t+\tau)^{l / n}
$$

and in view of (2.13),

$$
\text { support of } x \rightarrow u(x, t) \text { is contained in the set } r \leqslant C_{2}\left(t+\tau_{2}\right)^{l / n}
$$

both constants $C_{1}, C_{2}$ are positive and independent of $t$. It follows that

$$
t+\tau \leqslant\left(C_{2} / C_{1}\right)^{n / l}\left(t+\tau_{2}\right) \text {, i.e., } \tau \leqslant C(t+1) \quad(C>0) .
$$

Thus, in computing $\sup L$ in (2.17) we may restrict ourselves to $(L, \tau) \in \tilde{\Sigma}_{t}$, where

$$
\tilde{\Sigma}_{t}=\Sigma_{t} \cap\left\{L_{1} \leqslant L \leqslant I^{m-1}, 0 \leqslant \tau \leqslant C(t+1)\right\} .
$$

Since $\tilde{\Sigma}_{t}$ is a compact set, it follows that

$$
\sup _{\tilde{\Sigma}_{t}} L=\max _{\tilde{\Sigma}_{t}} L=L^{*}
$$


with $\left(L^{*}, \tau^{*}\right) \in \tilde{\Sigma}_{t}$ rendering the maximum; this completes the proof.

LEMMA 3.2. $L(t)$ is monotone decreasing in $t$, and there exists a sequence $t_{j} \uparrow \infty$ such that

$$
L\left(t_{j}\right)<L\left(t_{j+1}\right) \text { for all } j .
$$

Proof. Since (2.13) for a fixed $t$ implies the same inequality for $t$ replaced by $t^{\prime}$, $t^{\prime}>t$, it follows that $L\left(t^{\prime}\right) \leqslant L(t)$. To prove (3.3), we fix point $t=t_{0}$ and construct a point $t_{1}>t_{0}$ such that

this would establish (3.3).

$$
L\left(t_{0}\right)<L\left(t_{1}\right)
$$

We have

$$
u\left(x, t_{0}\right) \geqslant V_{L}\left(r, t_{0}+\tau\right) \quad \text { where } L=L\left(t_{0}\right) .
$$

We claim: there exists a $\xi>0$ such that

$$
u\left(x, t_{0}+\xi\right) \not \equiv V_{L}\left(r, t_{0}+\xi+\tau\right) \text { for } r<(\beta / c) L^{l / n}\left(t_{0}+\tau+\xi\right)^{l / n} .
$$

Indeed, otherwise

$$
u(x, t) \equiv V_{L}(r, t+\tau) \quad \text { whenever } t \geqslant t_{0}, V_{L}>0 .
$$

Recalling (2.15) we conclude that, for some $x^{0} \in R^{n}, \delta>0$,

$$
u\left(x, t_{0}\right)>0, V_{L}\left(r, t_{0}+\tau\right)=0 \text { if }\left|x-x^{0}\right|<\delta .
$$

But then

$$
u\left(x, t_{0}\right)>V_{L_{*}}\left(\left|x-x^{0}\right|, t_{0}+\tau_{*}\right)
$$

for some $L_{*}>0, \tau_{*}>0$.

Choose now a point $(\hat{x}, \hat{t})$ such that

$$
|\hat{x}|=(\beta / c) L^{l / n}(\hat{t}+\tau)^{l / n}
$$

(i.e., $\hat{x}$ lies on the boundary of the support $x \rightarrow V_{L}(r, \hat{t}+\tau)$ ) and

$$
\left|\hat{x}-x^{0}\right|<(\beta / c) L^{l / n}\left(\hat{t}+\tau_{*}\right)^{l / n}
$$

(i.e., $V_{L_{*}}\left(\left|\hat{x}-x^{0}\right|, \hat{t}+\tau_{*}\right)>0$ ). By (3.8) and comparison we then deduce that $u(\hat{x}, \hat{t})>0$, whereas from (3.7) we get that $u(\hat{x}, \hat{t})=0$, a contradiction.

Having proved (3.6) we can now write

$$
u\left(x, t_{0}+\xi\right) \neq V_{L}\left(r, t_{0}+\xi+\tau\right) \quad \text { if } r<(\tilde{\theta} \beta / c) L^{l / n}\left(t_{0}+\tau+\xi\right)^{l / n} \equiv r_{1}
$$

if $0<\tilde{\theta}<1,1-\tilde{\theta}$ sufficiently small.

We shall compare $u(x, t+\xi)$ with $V_{L}(r, t+\tau+\xi)$ for $r\left\langle r_{1}, t\right\rangle t_{0}$. Notice that

$$
V_{L}(r, t+\tau)>0 \text { if } r<r_{1}
$$

hence also $u$ is positive. We therefore have, in the classical sense,

$$
u_{t}=\Delta u^{m}, \quad\left(V_{L}\right)_{t}=\Delta\left(V_{L}\right)^{m} \text {. }
$$

It is easily seen that the function $w=u-V_{L}$ then satisfies in the cylinder, $r<r_{1}$, $t>t_{0}$

$$
w_{t}=a \Delta w+\sum b_{i} w_{x_{i}}+c w
$$


for some smooth coefficients $a, b_{i}, c ; a>0$. Since $w \geqslant 0$ in the cylinder, the strong maximum principle implies that either

$$
w(x, t)>0 \text { if }|x|<r_{1}, t>t_{0},
$$

or else $w \equiv 0$; the second possibility is ruled out by (3.9). Thus, for any $0<\theta_{1}<1$ and for any $\eta>\xi(\eta-\xi$ small enough)

$$
u\left(x, t_{0}+\eta\right)>V_{L}\left(r, t_{0}+\tau+\eta\right) \text { if } r<\theta_{1} \cdot(\beta / c) L^{l / n}\left(t_{0}+\tau+\eta\right)^{l / n} .
$$

By Lemma 2.1,

$V_{L}\left(r, t_{0}+\tau+\eta\right)>V_{L}\left(r, t_{0}+\tau+\eta-\varepsilon\right)$ if $r>\theta_{\varepsilon} \cdot(\beta / c) L^{l / n}\left(t_{0}+\tau+\eta\right)^{l / n}$ where $\theta_{\varepsilon} \rightarrow \theta_{0}, 0<\theta_{0}<1$. On the other hand,

$$
\left|V_{L}\left(r, t_{0}+\tau+\eta\right)-V_{L}\left(r, t_{0}+\tau+\eta-\varepsilon\right)\right|=o(1) \rightarrow 0 \text { if } \varepsilon \rightarrow 0,
$$

uniformly in $r$. Taking $\theta_{1}>\theta_{0}$ and choosing $\varepsilon$ sufficiently small (depending on $\xi, \eta$ ) we conclude, using (3.11), that

$$
u\left(x, t_{0}+\eta\right)>V_{L}\left(x, t_{0}+\tau+\eta-\varepsilon\right)
$$

in a region $r<R$ containing the support of the function

$$
r \rightarrow V_{L}\left(r, t_{0}+\tau+\eta-\varepsilon\right) .
$$

But then, if $L^{\prime}>L$ and $L^{\prime}-L$ is sufficiently small,

$$
u\left(x, t_{0}+\eta\right)>V_{L^{\prime}}\left(r, t_{0}+\tau+\eta-\varepsilon\right) .
$$

It follows that

$$
L\left(t_{0}+\eta\right) \geqslant L^{\prime}>L=L\left(t_{0}\right)
$$

Setting $t_{1}=t_{0}+\eta$, (3.4) follows.

4. Proof of Theorem 1.1 for $u_{0}$ having compact support. Consider the one-parameter family of functions

$$
u_{k}(x, t)=k^{n} u\left(k x, k^{n / l} t\right), \quad k \geqslant 1 .
$$

From (2.13) we deduce that

$$
u_{k}(x, t) \leqslant k^{n} V_{L_{2}}\left(k x, k^{n / l} t+\tau_{2}\right)=V_{L_{2}}\left(r, t+\tau_{2} / k^{n / l}\right) .
$$

Therefore, for any $\delta>0$,

$$
u_{k}(x, t) \leqslant C_{\delta} \quad\left(x \in R^{n}, t \geqslant \delta, k \geqslant 1\right)
$$

where $C_{\delta}$ is a constant depending on $\delta$.

Applying the continuity result of Caffarelli and Friedman [5], [6] we deduce that

the $u_{k}(k, t)$ are equicontinuous in $(x, t) \in R^{n} \times[\delta, \infty), \quad$ for $k \geqslant 1$.

Hence from any sequence $k_{i}^{*} \uparrow \infty$ we can extract a subsequence $k_{i}$ such that, for any $\delta>0$,

$$
u_{k_{i}}(x, t) \rightarrow w(x, t) \text { uniformly in }(x, t) \text { in compact subsets of } R^{n} \times[\delta, \infty) ;
$$

$w$, the limit function, may a priori depend on the sequence. 
By the energy inequality [14], [9] for (1.1),

$$
\int_{\delta}^{T} \int_{R^{n}}\left|\nabla\left(u_{k}\right)^{m}\right|^{2} d x d t \leqslant C \quad(C \text { constant })
$$

Hence,

$$
\nabla u_{k_{i}} \rightarrow \nabla w \text { weakly in } L_{\text {loc }}^{2}\left[R^{n} \times(0, \infty)\right] .
$$

Since each $u_{k_{i}}$ is a generalized solution, we deduce that also $w$ is a generalized solution.

Define

$$
L_{0}=\lim _{t \rightarrow \infty} L(t)
$$

We have

$$
u_{k}(x, t)=k^{n} u\left(k x, k^{n / l} t\right)
$$

and

$$
u(x, t) \geqslant V_{L(t)}\left(r, t+\tau_{t}\right), \quad \tau_{t} \leqslant C t(t \geqslant 1) .
$$

Hence

$$
u_{k}(x, t) \geqslant k^{n} V_{L\left(k^{n / t}\right)}\left(k r, k^{n / l} t+\tau_{k, t}\right)
$$

where

$$
\tau_{k, t} \leqslant \frac{1}{k^{n / l}} \tau_{k^{n / 4}} \leqslant C t .
$$

For each $t$ there is a subsequence $k_{i^{\prime}}$ of $k_{i}$ for which

$$
\tau_{k_{i}, t} \rightarrow \tilde{\tau}_{t}, \quad \tilde{\tau}_{t} \leqslant C .
$$

Taking $k=k_{i^{\prime}} \rightarrow \infty$ in (4.8) and using (4.5), (4.7), we get

$$
w(x, t) \geqslant V_{L_{0}}\left(r, t+\tilde{\tau}_{t}\right) .
$$

Lemma 4.1. For any $t>0$,

$$
w(x, t)=V_{L_{0}}\left(r, t+\tilde{\tau}_{t}\right) .
$$

Proof. Suppose otherwise; then for some $t>0$,

$$
w(x, t) \not V_{L_{0}}\left(r, t+\tilde{\tau}_{t}\right) \text { for } x \in R^{n} .
$$

Proceeding as in Lemma 3.2 (recall that $w$ is a solution of (1.1)) we find that for some $\eta>0$ and for sufficiently small $\varepsilon, 0<\varepsilon<\eta$,

$$
w(x, t+\eta)>V_{\hat{L}}\left(r, t+\tilde{\tau}_{t}+\eta-\varepsilon\right) \text { on supp } V_{\hat{L}},
$$

for some $\hat{L}>L_{0}$. Recalling (4.5) we deduce that

$$
u_{k_{i}}(x, t+\eta) \geqslant V_{\hat{L}}\left(r, t+\tilde{\tau}_{t}+\eta-\varepsilon\right)
$$

if $k_{i}$ is sufficiently large. Hence

$$
L\left(k_{i}^{n / l}(t+\eta)\right) \geqslant \hat{L}
$$

and, consequently, also $L_{0} \geqslant \hat{L}$, a contradiction. 
LEMMA 4.2. $\tilde{\tau}_{t}=0$.

Proof. Since $w$ is a generalized solution, the same is true of the function

$$
(x, t) \rightarrow V_{L_{0}}\left(r, t+\tilde{\tau}_{t}\right)
$$

Since also

$$
(x, t) \rightarrow V_{L_{0}}\left(r, t+\tilde{\tau}_{\delta}\right) \quad(\delta>0)
$$

is a solution, and both solutions agree on $t=\delta$, it follows that they agree for all $t \geqslant \delta$. Hence $\tilde{\tau}_{t}=\tilde{\tau}_{\delta}$ if $t \geqslant \delta$; thus $\tilde{\tau}_{t}=$ const $=\tau^{*}$.

Next, by (2.10),

$$
\begin{aligned}
u_{k}(x, t) & =k^{n} u\left(k x, k^{n / l} t\right) \geqslant k^{n} V_{L_{1}}\left(k r, k^{n / l} t+\tau_{1}\right) \\
& =V_{L_{1}}\left(r, t+\tau_{1} / k^{n / l}\right) .
\end{aligned}
$$

Taking $x=0, t=\delta$ we get

$$
u_{k}(0, \delta) \geqslant V_{L_{1}}\left(0, \delta+\tau_{1} / k^{n / l}\right) \geqslant \beta^{2 /(m-1)} / 2 \delta^{l}
$$

if $k$ is sufficiently large. On the other hand

$$
u_{k_{i}}(0, \delta) \rightarrow w(0, \delta)=V_{L_{0}}\left(0, \delta+\tau^{*}\right) .
$$

Therefore

$$
V_{L_{0}}\left(0, \delta+\tau^{*}\right) \geqslant \beta^{2 /(m-1)} / 2 \delta^{l} .
$$

Taking $\delta \rightarrow 0$ we deduce that $\tau^{*}$ must be equal to zero.

We have proved so far that

$$
u_{k_{i}}(x, t) \rightarrow V_{L_{0}}(r, t)
$$

uniformly in $(x, t)$ in compact subsets of $R^{n} \times(0, \infty)$. Since the supports of the functions

$$
x \rightarrow u_{k_{i}}(x, t)
$$

are uniformly bounded, by (2.13), we conclude that

$$
\int_{R^{n}} u_{k_{i}}(x, t) d x \rightarrow \int_{R^{n}} V_{L_{0}}(r, t) d x
$$

Observing that

$$
\int_{R^{n}} u_{k}(x, t) d x=k^{n} \int_{R^{n}} u\left(k x, k^{n / l} t\right) d x=I,
$$

the assertion (1.9) follows. Thus the limit in (4.11) is independent of the sequence $k_{i}^{*}$ that we have started with (just before (4.5)). It follows that

$$
u_{k}(x, t) \rightarrow V_{L_{0}}(r, t) \text { as } k \rightarrow \infty,
$$

where the convergence is uniform in $(x, t)$ in compact subsets of $R^{n} \times(0, \infty)$; in view of (2.13), the convergence is in fact uniform in $(x, t) \in R^{n} \times[\delta, \infty)$, for any $\delta>0$.

Taking $t=1$ in (4.12) we deduce that, as $k \rightarrow \infty$,

$$
k^{n} u\left(k x, k^{n / l}\right)-V_{L_{0}}(r, 1) \rightarrow 0 \text { uniformly in } x \in R^{n}
$$


or

$$
k^{n}\left[u\left(k x, k^{n / l}\right)-V_{L_{0}}\left(k r, k^{n / l}\right)\right] \rightarrow 0
$$

uniformly in $x \in R^{n}$. Replacing $k x$ by $x$ and setting $t=k^{n / l}$, the assertion (1.8) follows. This completes the proof of Theorem 1.1 in case $u_{0}$ has compact support.

5. Proof of Theorem 1.1. We shall now remove the restriction (2.11). The following estimate due to Benilan [3] and Veron [15] will be needed:

LEMMA 5.1. There exists a positive constant $C$ such that, for any nonnegative initial data $u_{0}$ in $L^{1}\left(R^{n}\right)$,

$$
\sup _{x \in R^{n}} u(x, t) \leqslant \frac{C}{t^{l}}\left[\int u_{0}(x) d x\right]^{2 l / n}
$$

For any $N=1,2, \ldots$, let $u_{0}^{N}(x)$ be an initial data satisfying (1.3), such that

$$
\begin{aligned}
& u_{0}^{N}(x)=u_{0}(x) \quad \text { if }|x|<N, \\
& u_{0}^{N}(x)=0 \quad \text { if }|x|>N+1, \\
& u_{0}^{N}(x) \leqslant u_{0}(x) \text { if } N \leqslant x \leqslant N+1 .
\end{aligned}
$$

Denote by $u^{N}(x, t)$ the solution of (1.1), (1.2) corresponding to $u_{0}^{N}$ and set

$$
\begin{aligned}
u_{k}^{N}(x, t) & =k^{n} u^{N}\left(k x, k^{n / l} t\right), \\
I_{N} & =\int_{R^{n}} u_{0}^{N}(x) d x .
\end{aligned}
$$

Then $I_{N} \rightarrow I$.

By Lemma 5.1,

$$
u_{k}(x, t) \leqslant\left(C / t^{l}\right) I^{2 l / n}
$$

Hence, by [5], [6], the $u_{k}$ are equicontinuous in compact subsets of $R^{n} \times(0, \infty)$. It follows that for any given sequence $k_{i}^{*} \uparrow \infty$ there exists a subsequence $k_{i}$ such that

$$
u_{k_{i}}(x, t) \rightarrow w(x, t) \text { uniformly in compact subsets of } R^{n} \times(0, \infty) ;
$$

the function $w$ may a priori depend on the sequence.

By what we have proved in $\$ 4$, for any $N$,

$$
u_{k}^{N}(x, t) \rightarrow V_{L_{N}}(r, t)
$$

uniformly in compact subsets of $R^{n} \times(0, \infty)$, where

$$
L_{N}=\left(I_{N}\right)^{m-1}
$$

Since $u \geqslant u^{N}$, we also have $u_{k} \geqslant u_{k}^{N}$, so that

$$
w(x, t) \geqslant V_{L_{N}}(r, t) \text {. }
$$

Taking $N \rightarrow \infty$ we find that

$$
w(x, t) \geqslant V_{L_{0}}(r, t)
$$

where $L_{0}$ is defined by (1.9). 
From (5.3) we obtain, using Fatou's lemma,

$$
\int_{R^{n}} w(x, t) d x \leqslant \lim \int_{R^{n}} u_{k_{i}}(x, t) d x=I
$$

consequently,

$$
\int_{R^{n}}\left[w(x, t)-V_{L_{0}}(r, t)\right] d x \leqslant I-L_{0}^{m-1}=0 .
$$

Comparing with (5.4) it follows that

$$
w(x, t)=V_{L_{0}}(r, t) .
$$

We deduce that the entire family $u_{k}$ is convergent to the same limit function, namely $V_{L_{0}}$.

We have thus proved that

$$
u_{k}(x, t)=k^{n} u\left(k x, k^{n / l} t\right) \rightarrow V_{L_{0}}(r, t)
$$

uniformly in compact subsets of $R^{n} \times(0, \infty)$.

Choosing $t=1$ and replacing $k x$ by $x$, the assertion (1.8) follows.

REMARK 1. The method of proof of Theorem 1.1 can be used to prove similar results for other equations. We illustrate this in the case of the heat equation

$$
u_{t}=\Delta u \text { in } R^{n} \times(0, \infty)
$$

with the Cauchy data

$$
u(x, 0)=u_{0}(x) \quad\left(x \in R^{n}\right)
$$

assuming that

$$
\begin{gathered}
u_{0}(x) \text { is continuous, } u_{0}(x) \neq 0, \\
0 \leqslant u_{0}(x) \leqslant C_{0} e^{-\alpha|x|^{2}} \quad\left(\text { for some } C_{0}>0, \alpha>0\right) .
\end{gathered}
$$

We shall show that, as $t \rightarrow \infty$,

$$
t^{n / 2}\left[u(x, t)-\frac{L_{0}}{(4 \pi t)^{n / 2}} e^{-|x|^{2} / 4 t}\right] \rightarrow 0
$$

uniformly in $x,|x|^{2} \leqslant C t$ (for any $C>0$ ), where

$$
L_{0}=\int_{R^{n}} u_{0}(x) d x
$$

(This result, for any $u_{0} \in L^{1}\left(R^{n}\right)$, can of course be proved directly from the formula

$$
\left.u(x, t)=\frac{1}{(4 \pi t)^{n / 2}} \int_{R^{n}} e^{-|x-y|^{2} / 4 t} u_{0}(y) d y .\right)
$$

We define

$$
V_{L}(r, t)=\frac{L}{(4 \pi t)^{n / 2}} e^{-r^{2} /(4 t)}
$$

and set

$$
L(t)=\inf _{(L, \tau) \in \Sigma_{t}} L
$$


where $\Sigma_{t}$ consists of all pairs $(L, \tau)$ such that $L \geqslant 0, \tau \geqslant 0$,

$$
u(x, t) \leqslant V_{L}(r, t+\tau) \quad\left(x \in R^{n}\right) .
$$

Then $L(t)$ is strictly increasing (assuming (2.15)); we use here an analog of Lemma 2.1 , namely, if

$$
V_{L}(r, \tau+\varepsilon)=V_{L}(r, \tau)
$$

then

$$
r=r_{\varepsilon} \rightarrow(2 n \tau)^{1 / 2} \text { as } \varepsilon \rightarrow 0 .
$$

Representing $u(x, t)$ in terms of the fundamental solution and the initial data, we find that

$$
u(x, t) \geqslant(c / t) e^{-\beta|x|^{2}} \quad \text { if } t \geqslant 1 \quad(c>0, \beta>0) .
$$

We now define

$$
u_{k}(x, t)=k^{n} u\left(k x, k^{2} t\right)
$$

and continue as in the preceding proof.

REMARK 2. For the equation (1.1) with $(n-2) / n<m<1$ the assertion (1.5) is still valid. Furthermore, P. Benilan (oral communication) proved a uniform Lipschitz continuity in any interval $(\delta, \infty), \delta>0$, with coefficient depending on $C$, where $\left\|u_{0}\right\|_{L_{1}} \leqslant C$. Defining $V_{L}(r, t)$ as in (1.6), but with $c^{2}$ replaced by $-c^{2}$, we see that the support of $x \rightarrow V_{L}(r, t)$ is all of $R^{n}$. The method of Remark 1 extends with minor changes to the present case, showing that (1.8) is valid.

The same procedure can be applied to nonlinear parabolic equations, such as

$$
u_{t}=\alpha u_{x x}+\beta\left|u_{x x}\right| \quad(\alpha>|\beta|>0)
$$

studied in [8], [2]; similarity solutions are constructed in [2].

\section{REFERENCES}

1. D. G. Aronson and L. A. Peletier, Large time behavior of solutions of the porous medium equation in bounded domains (to appear).

2. G. I. Barenblatt, Similarity, automodelling, intermediate asymptotics; theory and applications to geophysical hydro-dynamics, Gidrometeoizdat, Leningrad, 1978; English transl., Similarity, self-similarity and intermediate asymptotics, Consultants Bureau, New York and London, 1979.

3. $\mathrm{Ph}$. Benilan, Opérateurs accretifs et semigroupes dans les espaces $L^{p}(1<p<\infty)$ (to appear).

4. H. Brezis and M. G. Crandall, Uniqueness of solutions of the initial-value problem for $u_{t}-\Delta \varphi(u)$ (to appear).

5. L. A. Caffarelli and A. Friedman, Continuity of the density of a gas flow in a porous medium, Trans. Amer. Math. Soc. 252 (1979), 99-113.

6. __ Regularity of the free boundary of a gas flow in an n-dimensional porous medium, Indiana Univ. Math. J. 29 (1980), 361-391.

7. C. J. Van Duyan and L. A. Peletier, Asymptotic behavior of solution of nonlinear diffusion equation, Arch. Rational Mech. Anal. 65 (1977), 363-377.

8. S. Kamenomostkaya, On a problem in the theory of filtration, Dokl. Akad. Nauk SSSR 116 (1957), 18-20.

9. 76-87. , The asymptotic behavior of the solution of the filtration equation, Israel J. Math. 14 (1973),

10. S. Kamin, Similar solutions and the asymptotics of filtration equations, Arch. Rational Mech. Anal. 60 (1976), 171-183.

11. O. A. Oleinik, On some degenerate quasilinear parabolic equations, Seminari dell' Istituto Nazionale di Alta Matematica 1962-1963, Oderisi, Gubbio, 1964, pp. 355-371. 
12. L. A. Peletier, Asymptotic behavior of the solutions of the porous media equation, SIAM J. Appl. Math. 21 (1971), 542-551.

13. R. E. Prattle, Diffusion from an instantaneous point source with concentration-dependent coefficient, Quart. J. Mech. Appl. Math. 12 (1959), 407-409.

14. E. S. Sabinina, On the Cauchy problem for the equation of non-stationary gas filtration in several space variables, Dokl. Akad. Nauk SSSR 136 (1961), $1034-1037$.

15. L. Veron, Coercivité et propriétés regularisantes des semi-groupes non linéaires dans les espaces de Banach (to appear).

Department of Mathematics, Northwestern University, Evanston, IlunNois 60201

Department of Mathematics, Tel Aviv University, Tel Aviv, Israel 